\title{
Gesetz, Politik und Erzählung DER GastlichKeit
}

\author{
Evi FOUNTOULAKIS / BORIS PREVIŠIĆ
}

\section{Gastlichkeit}

In Heinrich von Kleists Erzählung »Die Verlobung in St. Domingo« (1811) bittet ein »Fremder«, Gustav von der Ried, inmitten der Haitianischen Revolutionskämpfe zwischen Einheimischen und Kolonialherren im Hause Congo Hoangos um Unterschlupf. Der fremde Gast wird zwar freundlich aufgenommen, aber dahinter verbirgt sich der perfide Plan, ihn bis zur Rückkehr des Hausherrn festzuhalten, der ihn zweifellos töten wird. Denn im gegenwärtigen »Kampfe gegen die Weißen « ${ }^{1}$ gehört die weiße Bevölkerung selbst zu den Opfern des Kolonialerbes. Zunächst scheint die Unheimlichkeit des Verrats nur der Sphäre der Gastgeber anzugehören; sie sind diejenigen, welche - nicht zum ersten Mal - eine Intrige inszenieren. Gustav von der Ried, der inmitten der Revolutionswirren um Zuflucht ersucht, ist nicht nur ein Gast, sondern aufgrund seiner Hautfarbe ein Fremder und, mehr noch, der Feind, denn im rechtlichen Ausnahmezustand der Revolution ist die Verletzung der nominellen Machtordnung, scheint der Verrat an den Gesetzen des Gastlichkeit bereits vorweggenommen. Das gilt nicht nur für die Rechtsgesetze, sondern im Umfeld Congo Hoangos auch für das Gesetz der Gastfreundschaft: ${ }^{2}$ So hat der vorübergehend abwesende Hausherr befohlen, alle Weißen vorgeblich als Gäste, tatsächlich jedoch als Geiseln festzuhalten. Gustav wiegt sich aufgrund der vermeintlich geltenden Gesetze der Gastfreundschaft, welche die (in Umwälzung befindlichen) rechtlichen Kodifizierungen und sozialen Konventionen der Gastlichkeit umfassen, in falscher Sicherheit. Die ritualhafte Einhaltung gastlicher Konventionen in der Form der Begrüßung, Bewirtung, Erzählung der eigenen Herkunft usw. bildet denn auch den strukturellen Rahmen der Handlungsereignisse.

1 Heinrich von Kleist: »Die Verlobung in St. Domingo«, Sämtliche Werke, Bd. II/4, S. 9.

2 Zur Unterscheidung zwischen >Gesetz< und >Gesetzen der Gastfreundschaft< s. Kap. 3 dieser Einleitung. 
Der fremde Gast scheint lange Zeit nichts von der ihm drohenden Gefahr der vordergründig mitfühlenden Gastgeber zu ahnen und wähnt sich bei seiner Gastgeberin Babekan und ihrer Tochter Toni gar in der Gesellschaft solidarischer Leidensgenossinnen. ${ }^{3}$ Doch die Pläne der Gastgeber werden mehrfach durchkreuzt, denn das ungeschriebene Gesetz der Gastfreundschaft verlangt einerseits, niemanden abzuweisen oder zu versehren, der um Schutz und Aufnahme bittet, andererseits aber auch, den Gast nicht gänzlich zu integrieren, da er sonst seiner Andersheit und seines Status als Gast verlustig ginge. ${ }^{4}$ Dieses grundlegende Gesetz wird von beiden Seiten, von Gast und Gastgebern, gebrochen; Congo Hoango und Babekan hintergehen es durch die heimliche Geiselnahme (bzw. Sequestrierung) des Fremden, während Toni und Gustav es durch die titelgebende »Verlobung « nicht nur in eine versuchte Integration wandeln; sie hypostasieren die Unheimlichkeit des Verrats auch auf Seiten des Gastes: Tonis Absicht, den fremden Gast zu retten, wird von diesem verkannt, so dass er sie schließlich aufgrund der von ihm vermuteten Täuschung tötet und sich, nachdem er seinen Irrtum erkannt hat, selbst richtet. Der Gast unterminiert sowohl die gegenwärtigen Landes- bzw. Kriegsgesetze wie auch den verbrecherischen Plan des Hausherrn, welche beide die Exklusion - bis hin zur Elimination - aller Weißen vorsehen, durch seine heimliche Verlobung. Doch indem Gustav sich offenkundig einer bedingten Form von Gastfreundschaft ausgesetzt wähnt, muss ihm die von der Gastgeberin Toni ${ }^{5}$ geübte absolute bzw. unbedingte Gastfreundschaft unbewusst bleiben; den Versuch, die Aufnahme dieses Fremden, wo er nicht angenommen werden kann, d.h. wo das Gesetz ihn nicht als Gast vorsieht, zu ermöglichen und Gastfreundschaft außerhalb jeder Pflicht, Ökonomie und jeden Kalküls zu üben, bezahlt Toni mit dem Leben. ${ }^{6}$

Der Ausnahmezustand des Gastes, der dem Alltagsdasein enthoben ist und andere des Alltagsdaseins enthebt, kulminiert hier im Ereignis, weil er nicht als geladener Gast erscheint, auf dessen Ankunft man vorbereitet und den zu empfangen man in der Lage ist. ${ }^{7}$ Die Unbedingtheit

3 »Freunde der Weißen, die selbst viel der Parthei wegen, die sie ergriffen, von den Schwarzen leiden müßten, hätten ihn in ihrem Hause mitleidig aufgenommen.« (»Die Verlobung in St. Domingo«, Sämtliche Werke, Bd. II/4, S. 56).

4 Vgl. Simon: »Ikononarratologie«, S. 307.

5 In der Chronologie der Erzählung etwa seit jener berühmten Ellipse, welche die Verlobung markiert: »Was weiter erfolgte, brauchen wir nicht zu melden, weil es jeder, der an diese Stelle kommt, von selbst lies’t.« (»Die Verlobung in St. Domingo«, Sämtliche Werke, Bd. II/4, S. 43). Bereits hier übertritt Toni die ihrer intriganten Gastgeberinnenrolle vorgegebene Grenze, »den Fremden keine Liebkosung zu versagen, bis auf die letzte, die ihr bei Todesstrafe verboten war« (ebd., S. 10).

6 Zum Begriff der >Gabe<vgl. Derrida: Falschgeld und Bahr: Die Befremdlichkeit des Gastes, S. 24-26.

7 Vgl. Derrida: Eine gewisse unmögliche Möglichkeit, S. 33. 
der Aufnahme des in der Erzählung > unmöglichen< Gastes ${ }^{8}$ unterläuft die sittlichen Gesetze der Gastlichkeit, welche mit der konventionalisierten Aufnahme des Gastes bestimmte Regeln vorschreiben bzw. perpetuieren, damit aber zugleich die Möglichkeit bestimmter Ausschlüsse implizieren, die sie selbst zu ungastlichen Gesetzen bzw. Gesetzen der Ungastlichkeit machen. ${ }^{9}$ Denn die $»$ strukturierte Vielfalt $«{ }^{10}$ der exkludierenden Gastgesetze ist das Ergebnis sozialer, politischer und historischer Differenzierungen und somit nicht universell zu denken. Bis zur Entstehung der Territorialstaaten galt die gastliche Aufnahme als eine zirkulierende »>Freigiebigkeit< in einem vagen, undeterminierten Tauschsystem «, doch wurde immer wieder darauf verwiesen, dass der Umgang mit dem Gast »historisch wie ethisch allen auf Tausch beruhenden und in Äquivalenzen rechnenden Verhältnissen voraus « liege. ${ }^{11}$ Die unbedingte Gastlichkeit - das als anthropologisches Grundrecht zu verstehende Gesetz der Gastlichkeit - zeigt allerdings bereits in der Antike mit »Symbolon« und »Tessara« (Gastmarken) erste Züge einer Institutionalisierung, die seit der Zeit Karl des Großen mit den Gesetzen der Gastfreundschaft als zunehmend bedingte, begrenzende und ausgrenzende Rechte kodifiziert wurde. Die Gastfreundschaft - das Recht auf gastliche Aufnahme desjenigen, der unterwegs ist - erfährt somit eine Wandlung in ein zunächst ungeschriebenes, dann national geregeltes, restriktives Gast- bzw. Fremdenrecht, womit die »Geburt des Fremden« zugleich die »Verabschieb/ dung des Gastes « markiert. ${ }^{12}$

8 Zum Begriff des Unmöglichen vgl. ebd.

9 Bahr: Die Sprache des Gastes, S. 251. Heute ließe sich in diesem Zusammenhang vielleicht eher von >Geboten< der Gastfreundschaft sprechen, um die unterschiedliche Sanktionsfähigkeit zu verdeutlichen. Die Unterscheidung zwischen Gesetz und Gebot würde eine historische Zäsur markieren: Das Gastrecht lässt sich etwa bis zum Mittelalter als ein positivierbares Recht verstehen, denn das ius hospitalis wird mit der Entstehung der Nationalstaaten zur Zeit des Absolutismus von einem Fremden- bzw. Ausländerrecht abgelöst und findet seine >Fortsetzung< am ehesten im allgemeinen Menschenrecht des 19. Jh. (vgl. Bahr: Die Sprache des Gastes, S. 36 und Kap. 6). Gegenwärtig lässt sich nicht mehr von einem positivierbaren Gastrecht sprechen; für die fremden Gäste gilt das Ausländerrecht. Die Gesetze der Gastlichkeit sind nur noch als Teil der Sitte (wofür der Begriff >Gebot< zutreffender erscheint) zu betrachten. Es müsste also für die Neuzeit darum gehen, die Gesetze der Gastlichkeit in Bezug auf das Sittengesetz zu untersuchen.

10 Derrida: Von der Gastfreundschaft, S. 61.

11 Bahr: Die Sprache des Gastes, S. 237.

12 Ebd., S. 236. 


\section{Die Fremdheit des Gastes}

Nicht alles, was uns unbekannt ist, befremdet uns; es gibt Dinge, die uns unvertraut sind und uns dennoch unberührt lassen. In der Regel werden sie unbemerkt an uns vorüberziehen, sei es als unbekannte Person, zumal wenn sie uns inmitten einer Masse, z.B. auf der Straße, im Konzert oder in einem Geschäft begegnet, sei es als fremde Sprache oder Idee, die in uns nichts bewegt. Nur das, »was uns angeht, indem seine Selbstverständlichkeit zerbricht, das befremdet uns «. Indem das Fremde uns >anspricht<, indem es unsere Eigensphäre durchdringt, vermag es eine (Re)aktion auszulösen, deren Charakter vom Grad und der Art der Befremdlichkeit abhängt: Führt die Befremdung zu Angst und Unbehagen und wird als Störung oder Gefahr wahrgenommen, so ist mit Abwehr und Ablehnung zu rechnen, welche der >Überfremdung< zuvorkommen sollen. Eine solche >Fremdenfeindlichkeit $<$ kann auch in ihr Gegenteil umschlagen: wenn alle Hoffnung auf eine von außen kommende Entität gesetzt wird, die als Chance, Heilung oder Erlösung verstanden wird. Als mittlerer Weg zwischen Ausgrenzung und Integration galt dabei lange Zeit eine Duldsamkeit, die nicht mit Gleichgültigkeit zu verwechseln ist, und als deren herausragendstes Beispiel die Gastfreundschaft gelten kann. ${ }^{13}$

Doch die Fremdheit, welche der Gastbegegnung innewohnt, ist nicht allein durch eine Unkenntnis oder ein Außenstehen des Gastes zu erklären. Die Befremdlichkeit des Gastes besteht vielmehr darin, dass er jede Berechnung, mithin jeden Erwartungshorizont sprengt; es lässt sich schwerlich - bestenfalls hypothetisch - abschätzen, was er mit sich bringt und was er als sein Vermächtnis hinterlassen wird. Obschon man vielleicht seinen Namen und seine Herkunft kennt, sind zumindest seine Absicht, die Dauer sowie die Wirkung seines Aufenthalts nicht mit Sicherheit zu deuten, denn dem sprachlichen Bekenntnis liegt die Möglichkeit der Lüge zugrunde. Wer zu Gast ist, bringt Qualitäten in den Raum, der ihm eröffnet wird, welche nicht aus diesem selbst stammen; worin diese bestehen, und ob sie - um die Extreme zu betonen - heilsam, beglückend oder bedrohlich sind, ist nicht $\mathrm{zu}$ antizipieren. Der fremde Gast kann sich, wie Georg Simmel es im Hinblick auf den Fremden feststellt, durch »Beweglichkeit«, »Objektivität« und »Freiheit « auszeichnen »und ist in seiner Aktion nicht durch Gewöhnung, Pietät, Antezedentien gebunden $« .{ }^{14}$ Freier als der Ansässige, verfügt er über die Möglichkeit, objektiver zu urteilen. »[D]aß ihm oft die überraschendsten Offenheiten und Konfessionen, bis zu dem Charakter der Beichte, entgegengebracht werden, die man jedem Nahestehenden sorg-

13 Bahr: Die Befremdlichkeit des Gastes, S. $16 \mathrm{f}$. 
fältig vorenthält $\ll^{15}$, gilt auch bei Simmel nicht so sehr für den Fremden, als vielmehr für den »Weiterziehenden«, also denjenigen, der vorübergehend um Aufenthalt ersucht. Andererseits lässt sich nicht ausschließen, dass der Gast über eine kriminelle Vergangenheit oder über ebensolche Energien verfügt, dass man sich einem Betrüger, Mörder oder gar dem personifizierten Bösen ausgesetzt sieht. Als Meister der Darstellung unheimlicher Gasterzählungen kann E.T.A. Hoffmann gelten, welcher den verstörenden Einbruch des Gastes in zahlreichen Variationen schildert. ${ }^{16}$ Deutlich wird, dass der Gast durch seine multiple Uneinschätzbarkeit eine potenzielle Bedrohlichkeit in sich birgt, welche ihn unberechenbar und seine Gabe verunsichernd erscheinen lässt.

Die gastliche Begegnung ist nicht nur von einer spezifischen Fernnähe ${ }^{17}$, sondern auch vom Raum geprägt, welcher - ob wirtlich oder unwirtlich - Offenheit und somit Gastlichkeit gewährt. ${ }^{18}$ Die Bestimmungen des $\mathrm{Zu}$-Gast-Seins sind immer auch räumliche: Gerade, wenn kein personifizierter Gastgeber anwesend ist, gewährt mindestens der Raum gastlichen Aufenthalt an diesem Ort, unter dem Himmel, in der Welt. Darauf verweist Bahr mit der Prägung >gastliches Milieu<, und auch Derrida macht den Raum implizit zum Träger der Gastlichkeit, indem er auf der Notwendigkeit der Eröffnung eines Ortes, einer Stätte (lieu) für die Gastlichkeit insistiert. ${ }^{19}$

Neben dem Raum, welcher dem Gast zu seinem Erscheinen eröffnet werden muss, ist seine spezifische Zeitlichkeit als Grundformel zu verstehen: Das $\mathrm{Zu}-$ Gast-Sein ist ein vorübergehender, endlicher Zustand. Als Gast, der - geladen oder ungeladen - vorübergehend an einem Ort verweilt, kommt man zugleich in den eigenen Abschied hinein. Das Temporäre, die Unstetigkeit und das Zeitweilige markieren die zeitliche Grundstruktur der Gastlichkeit. Selbst wenn vom flüchtigen Gast, vom

15 Ebd., S. 767.

16 Vgl. zahlreiche der Novellen aus dem Zyklus der Nachtstücke, z.B. E.T.A. Hoffmann: »Der Sandmann«; ders.: »Ignaz Denner«; oder ders.: »Der unheimliche Gast « aus der Novellensammlung Die Serapions-Brïder.

17 Bereits Simmel sieht im »Exkurs über den Fremden« das Verhältnis des Fremden zu räumlich und sozial fixierten Personen durch ein spezifisches Verhältnis von Nähe und Ferne gekennzeichnet. Allerdings gilt die räumliche und soziale Fixierung für den Gastgeber nur bedingt; denn ein Rollentausch, ein Positionstausch zwischen Gast und Gastgeber liegt der gastlichen Begegnung zugrunde.

18 Dass Offenheit Gastlichkeit bedeutet, ist für Bahrs metaethischen Zugang das zentrale Argument: »Die Gastlichkeit [...] ist Metaethos: Gastlichkeit eines Gesetzes, das die Lücke im Gesetz zu wahren versteht, die dem anderen als Gast einen Platz freimacht, einen Weg eröffnet, ihn zur Sprache kommen lässt.« (Bahr: Die Befremdlichkeit des Gastes, S. 54).

19 Vgl. Derrida: Von der Gastfreundschaft, S. 27; Bahr: Die Anwesenheit des Gastes. Waldenfels untersucht die Topographie des Fremden hinlänglich in seinem gleichnamigen Werk. 
wiederkehrenden Stammgast oder vom lebenslänglichen Hausgast die Rede sein kann, so spricht das Gastsein als Existenzial unter dem Aspekt eines Ankommens in den Abschied von unserer Endlichkeit. Dauer und Endlichkeit lassen sich folglich in der Formel einer »Ankunft in den verweilenden Abschied « kondensieren. Die Gastlichkeit gibt sich nur als »vorhergehende und vorübergegangene ${ }^{20}$ und ist somit nur in der Nachträglichkeit erkennbar ${ }^{21}$, während der Gast »dem Vergangenen [gleicht], das nirgends anders zu finden ist als in seinen Nachwirkungen oder in der Erinnerung «, wie es Waldenfels im Falle des Fremden formuliert. ${ }^{22}$ Doch gerade die Nachträglichkeit führt dazu, dass der Gast selbst niemals als Ganzer fassbar wird. ${ }^{23}$ Die Unmöglichkeit seiner Verfestigung, seine räumliche und zeitliche Unfixierbarkeit als Voraussetzung des Gastseins verfügt dabei immer über das Potenzial, bedrohlich zu kippen. Die Beunruhigung, die aus dieser Unverfügbarkeit resultiert, setzt den Gast dem Versuch seiner Tilgung aus durch Festschreibung bzw. Normalisierung (Integration, Assimilation) oder durch Abwehr und Ausschluss.

Der temporäre, nur vorübergehend zugestandene Zustand des Gastes macht zugleich deutlich, dass es sich beim Gast weder um eine wesentliche, noch um eine zufällige Kategorie des Menschen handelt. So liefert Bahr zufolge das Gastsein auch aus nicht-anthropologischer Sicht ein neues Kategorienmodell: Zu Gast sein kann - das belegt die >Alltagssprache<genau so wie die Lyrik, Erzählungen oder Dramenrede - ein Mensch, eine Person, aber genauso eine Empfindung, eine Krankheit, ein Tier oder begriffliche Abstrakta wie z.B. die Natur u.v.m. ${ }^{24}$ Die Literatur verfügt über mannigfaltige Beispiele dieser Art, die nahelegen, dass die Anwendung des >Gastes $<$ auf andere Verhältnisse als zwischen zwei Personen eine sehr lange Tradition hat, die nicht einer subjektiven Haltung entspringt, sondern ein bislang nicht oft thematisiertes Phänomen darstellt. ${ }^{25}$ Folglich >ist< niemand Gast oder >ist nicht< Gast, denn jeder ist nur $z u$ Gast. ${ }^{26}$ Die Gastlichkeit bietet sich

20 Bahr: Die Befremdlichkeit des Gastes, S. 53.

21 Hier ist einer von zahlreichen Anschlüssen an die Gabe möglich, von der Derrida in Falschgeld auch aus der Perspektive der Nachträglichkeit spricht.

22 Waldenfels: Topographie des Fremden, S. 26.

23 Vgl. ebd., S. 51.

24 Die sprachliche Ubiquität am Beispiel der Lyrik untersucht detailliert der Beitrag von Hans-Dieter BAHR in diesem Band.

25 Diese Betrachtungsweise des Gastes bedeutet bzw. impliziert zugleich, dass der Mensch nicht die Substanz des Gastes darstellt, womit der Auffassung des Gastes als eines anthropologischen Fundamentals insofern widersprochen wird, als sie nur als ein beschränkter Diskurs - einer Diskurs unter vielen, wenn auch ein häufiger identifiziert wird. Ausführlich hergeleitet und begründet wird dies in Bahr: Die Anwesenheit des Gastes.

Vgl. Bahr: Die Befremdlichkeit des Gastes, S. 54. 
somit an als Kategorie, die die Klassifizierungen der abendländischen Logik durchbricht bzw. ein Modell für Phänomene des Dritten bietet. Wenn die Figur des Gastes das Potenzial zu einem neuen Wissenschaftsparadigma besitzt ${ }^{27}$ und das gastliche Verhältnis Anschlussmöglichkeiten über motivische Untersuchungen des Gastes hinaus schafft, muss womöglich die Reduktion des Gastes auf die Person und damit auf eine soziale Rolle in Frage gestellt werden, wie Bahr es in seiner metaethischen Untersuchung tut. Sein Begriff des Gastes als Figur des unassimilierbaren Dritten, der alle Oppositionen durchquert bzw. unterwandert, wofür er den Ausdruck >Transversalität< verwendet, ist somit universell und nicht etwa rein anthropologisch gedacht.

Bei genauerer Betrachtung lässt sich das Zu-Gast-Sein aus zweifacher Perspektive nicht mit den Kategorien der klassischen Logik fassen: Einerseits, da es weder als wesentliche Eigenschaft, noch als bloße Eigenart des Menschen, noch als nur zufällig und äußerlich gedacht wird; hiermit wird die Möglichkeit der Überschreitung der Anthropologie angedeutet. ${ }^{28}$ Andererseits, weil der Gast aus einer ethischen Perspektive als jene Figur gedacht werden kann, die aufgrund des universellen Gesetzes der Gastlichkeit weder verneint noch bejaht werden darf. In formalisierter Sprache lässt sich der Gast folglich als weder $a$ noch non a bestimmen. ${ }^{29}$ Als solcher aber stellt er eine logische Unmöglichkeit bei zugleich manifester Präsenz dar. Er ist eine paradoxe Figur des Dritten: eigentlich sollte es ihn nicht geben, es gibt ihn aber. Aus dieser aporetischen Situation resultieren gleichzeitig auch seine Unheimlichkeit und Unverfügbarkeit.

Nimmt man des Weiteren die sprachlich manifeste Vielfalt, ja Universalität des Gastes ernst, so erweist sich beispielsweise auch die Opposition von Gast und Gastgeber als künstlich. Sie entspricht zwar wahrgenommenen sozialen Rollen; doch darüber hinaus zeichnet sie sich gerade nicht durch eine Opposition aus, sondern durch die Möglichkeit der Supplementation, der Austauschbarkeit, die ihr innewohnt ${ }^{30}$, wie sie sich auch in gängigen sprachlichen Formeln wie »fühlen Sie sich wie zu Hause « manifestiert; ebenso wirkt sich die Temporalität des Gastes auf ein nur als temporär zu denkendes Gastgebersein aus. Darauf weist auch die Etymologie indoeuropäischer Gastbegriffe hin, welche neben der semantischen Ambiguität zwischen Fremdem und Gastfreund (gr. xénos), Fremdem und Feind (lat. hostis), bewirtetem Fremdem und Krieger (dt. Gast kennt bis ins Mittelhoch-

27 Vgl. Parr/Friedrich: »Von Gästen, Gastgebern und Parasiten«, S. 12f.

28 Vgl. Bahr: Die Befremdlichkeit des Gastes, S. 15, sowie ders.: Die Sprache des Gastes.

29 Vgl. Simon: »Ikononarratologie«, S. 307.

30 Vgl. Derrida: Von der Gastfreundschaft, S. 90. 
deutsche beide Bedeutungen $)^{31}$ auch eine semantische Oszillation zwischen Gast und Gastgeber kennzeichnet; das gilt für das frühe gr. xénos, für das lat. hospes ${ }^{32}$ wie auch für die zeitgenössische Terminologie, z.B. frz. hôte. Diese sprachliche Uneindeutigkeit nimmt bereits den Hinweis auf einen der Gastfreundschaft inhärenten Rollentausch vorweg. Deutete man die Begriffe Gast und Gastgeber als Oppositionen, läge es nahe, der Gastfreundschaft ein Tauschverhältnis zugrunde zu legen, wobei bewegliche Gaben zwischen den beiden stabilen Positionen zirkulierten. Die Instabilität der sozialen Rollen von Gast und Gastgeber jedoch verunmöglicht grundsätzlich einen solchen Tausch, was nicht bedeutet, dass er nicht stattfindet; aber er findet als Versuch statt, die Unberechenbarkeit des Gastes durch die Überführung in ein ökonomisches Kalkül zu tilgen. ${ }^{33}$ Der Gast löst folglich Tauschrituale aus, die der »Kompensation einer Angst vor dem Gast « dienen. ${ }^{34}$ Sie stellen den Versuch dar, den Gast aus seiner Paradoxie als dem Dritten zu lösen und ihn in eine stabile soziale Bindung zu zwingen. Dabei gilt es, den semantischen Ambivalenzen Rechnung zu tragen, welche die Tauschverhältnisse von der Objekt- auf die Subjektebene verlagern; in der Regel sind die Positionen der Tauschenden stabil, und ein Objekt zirkuliert als Gabe. Gast und Gastgeber hingegen befinden sich in einem instabilen Verhältnis und tauschen selbst die Rollen; von einem Tausch oder einer Oszillation kann also v.a. in Bezug auf ihr jeweiliges Verhältnis selbst gesprochen werden: Es ist ein wechselseitiges SichZu-Gast-Werden.

Die Nähe zum Tausch und zur Ökonomie findet sich insbesondere in den Gesetzen der Gastfreundschaft: Wenn die (bedingten) Gesetze der Gastfreundschaft als Gesetze der Großzügigkeit aufgefasst werden, so zeigt die Figur des Gastes auf, dass gerade diese vermeintliche Großzügigkeit die (unbedingte) Gastfreundschaft verhindert - gerade, weil sie denjenigen $\mathrm{zu}>$ normalisieren $<$, $\mathrm{zu}$ berechnen, einzuordnen versucht, welchen gerade seine Unkalkulierbarkeit auszeichnet. Auch wenn man ihn zu kategorisieren versucht, sprengt er noch die Unterscheidung zwischen Fremdem und Eigenem. Der von außen kommende Gast markiert einen Einbruch in das vermeintlich Bekannte, das er dadurch zu entfremden vermag. Durch die Brille des so weit Entfernten, dass er als Gast wahrgenommen werden kann, betrachtet, gewinnen die bislang vertraut, bekannt, eigen, zugehörig, assimiliert scheinenden Dinge eine neue Qualität, die sie uns entzieht, entfremdet, unheimlich macht und uns somit ihre eigentliche Unverfügbarkeit aufzeigt (die sich zuvor

31 Vgl. >Gast< im Etymologischen Wörterbuch des Deutschen, S. 400.

32 Vgl. Benveniste: Indoeuropäische Institutionen.

33 »Die Gastsemantik widersteht den Begriffen des Tausches und der Zirkulation, ihr Kern ist ein Inkompatibles und Inkommensurables, ein nicht Operationalisierbares.« (Simon: »Ikononarratologie«, S. 308). 
durch eine vielleicht allzu nahe Beziehung der Wahrnehmung entzogen hatte). Die Unverfügbarkeit des Gastes wirkt daher selbst unheimlich, potenziell beunruhigend oder verstörend. Doch besteht die disruptive Macht oder Gewalt des Gastes nicht nur darin, die Aufmerksamkeit auf die eigene Verdrängungsleistung zu richten. Die Befremdlichkeit des Gastes liegt vielmehr darin, dass uns unerwartet das Ungewöhnliche überfällt und uns plötzlich >anspricht $<-$ sie besteht darin, dass der Gast in Frage stellt, »und zwar gleichermaßen das Eigene wie das Andere, das ihm gegenübertritt «. Es handelt sich hierbei jedoch um eine offene Frage, die Möglichkeit des Fragens und somit des Fremdseins selbst, die sich angesichts des Gastes stellt. ${ }^{35}$

Was also bedeutet es, den Gast als Fremden $\mathrm{zu}$ betrachten? Zunächst ganz einfach, Figuren der Gastlichkeit aus der Perspektive des Fremden bzw. der Fremdheit zu untersuchen. Die Erfahrung des Fremden und des Befremdlichen kennt dabei bekanntermaßen zwei Extreme des Umgangs: die Ablehnung und Abwehr als Reaktion auf Unbehagen und Ängste, die das Befremdliche als störende, unheilvolle Gefahr zu tilgen versuchen; und die Aufnahme bis hin zur Assimilation oder Integration, die das Befremdliche durch Einverleibung gleichermaßen zum Verschwinden zu bringen drohen. ${ }^{36}$ Auf die etymologisch verbürgte Ambivalenz dieser Position - >hostis< in der Oszillation zwischen Gastfreundschaft und feindlicher Ausgrenzung - wurde bereits hingewiesen. Die Gastlichkeit als Dazwischen würde somit die Befremdlichkeit als offene Frage thematisieren, die ein Jenseits von Integration und Ausgrenzung zu denken ermöglicht.

$* * *$

Die vorliegenden Beiträge positionieren sich in erster Linie in ihrem Verhältnis zur Gastlichkeit und nehmen eine spezifische Betrachtungsweise ein, die die Fremdheit dieses Verhältnisses zum Kerngegenstand macht. Selbst da, wo das Fremde sich nicht augenfällig - insbesondere in der Personifizierung durch einen unbekannten, unerwarteten Gast präsentiert, ist seine Spur noch in einer Befremdlichkeit vorhanden. Diese Feststellung, die zahlreiche der versammelten Beiträge treffen, stärkt die (bislang v.a. von philosophischen Untersuchungen vorgeführte) These, wonach der Gast immer einen Einbruch markiert, der eine Veränderung bewirkt - eine Veränderung, die von der bloßen Objektivierung durch den Dritten zur Infragestellung des Eigenen, Irritation, potenziellen Verstörung bis hin zur existenziellen Verunsicherung reichen kann. ${ }^{37}$ Der Ausnahmezustand, in dem der Gast erscheint oder den er als > Vermächtnis $<$ hinterlässt, reicht in den untersuchten literarischen Texten im vorliegenden Band bezeichnenderweise

35 Bahr: Die Befremdlichkeit des Gastes, S. 18.

36 Vgl. ebd., S. 16f.

37 Vgl. z.B. Derrida: Von der Gastfreundschaft; ders.: Eine unmögliche Möglichkeit. 
vom Extremfall der Revolution (wie in Kleists »Die Verlobung in St. Domingo«) über die realistische Familienstiftungslegende (Stifters »Der Kuß von Sentze «) bis hin zu postmodernen Existenz- oder Identitätskrisen (Štiks' Archive der Nacht, Handkes Mein Jahr in der Niemandsbucht).

Die Beliebigkeit, in welcher der Gast zu verschwinden droht, wenn seine Rolle bloß als soziales Gewand verstanden wird, das jeder überziehen kann, oder als Leerstelle, die ein jeder einnehmen kann, droht auch auf eine Betrachtung der Gastlichkeit überzugreifen. ${ }^{38} \mathrm{Um}$ im vorliegenden Band, dessen Beiträge auf die Tagung unter dem Titel »Narrative Muster zwischen Gastfreundschaft und Ausgrenzung « an der Universität Basel (19.-21. November 2009) zurückgeht, der Gefahr einer solchen Begriffsverwässerung vorzubeugen, ist er nach einer thematischen Dreiteilung gegliedert, welche ihre Schwerpunkte bei der Frage nach dem Gesetz, der Politik und der Erzählung der Gastlichkeit setzt.

\section{Gesetz}

Die Unterscheidung zwischen bedingter und unbedingter Gastfreundschaft markiert bereits die beiden Pole, die der Gastlichkeit inhärent sind, einerseits als plurale Gesetze, andererseits als singuläres Gesetz, welches stärker als Ethos konnotiert und den konventionellen bzw. gesetzlichen Handlungsmaximen der Gastfreundschaft entgegengesetzt ist. Das Gesetz bricht zugleich mit den Gesetzen, d.h. dem Pakt der Gastfreundschaft als Recht oder Pflicht, bzw. versucht, die institutionalisierte Seite der Gastfreundschaft zu überwinden. ${ }^{39}$ Diese Form der unbedingten Gastfreundschaft ist also als »das Gesetz in seiner universellen Singularität ${ }^{40} \mathrm{zu}$ verstehen, das die kodifizierten Gastgesetze hyperbolisch zu überwinden (oder zu subvertieren?) versucht. Sie muss die bedingte Gastfreundschaft zwar nicht verdammen, aber sie ist ihr ebenso heterogen wie die Gerechtigkeit dem Recht, und doch genauso untrennbar mit ihr verbunden. ${ }^{41}$

Dennoch tritt der Gast, zumal als Fremder, nicht jenseits der Rahmung zum Vorschein, ob diese nun in sozialen Bedingungen, Institutionen, kodifizierten Gesetzen o.ä. besteht. Mit seinem Erscheinen wird

38 Auf die Gefahr dieser Beliebigkeit im Falle des Gastes als Universal wurde bereits hingewiesen in Parr/Friedrich: »Von Gästen, Gastgebern und Parasiten«, 9. Neben Renate Bürner-Kotzams Vertraute Gäste ist dies eine der wenigen Publikationen, die den Gast aus spezifisch literaturwissenschaftlicher Perspektive untersucht.

39 Derrida: Von der Gastfreundschaft, S. 27.

40 Ebd., S. 61.

41 Ebd., S. 28. Derrida führt das intrikate Verhältnis von Recht und Gerechtigkeit in Gesetzeskraft eindrücklich vor. 
der Gast unweigerlich in bereits existierende Muster eingeordnet; zwar mag er in die Ordnung einbrechen, doch fängt diese ihn im Bemühen auf, ihn zu normalisieren. Warum aber muss der Gast, warum die Gastlichkeit >normalisiert< werden? Vielleicht, weil der Gast derjenige ist, der den »Primat des Regelfalles ${ }^{42}$ in kompromisslosester Weise in Frage zu stellen vermag, so dass seine Stillstellung nur im Rückgriff auf Rituale, Traditionen und Bräuche ${ }^{43}$ (also auf Gesetze unterschiedlicher Ordnungsebenen mit abweichendem Sanktionspotential) möglich scheint, die seine befremdlichen Qualitäten aufzufangen, eben zu >normalisieren $<$ scheinen.

Doch inwiefern ist der Fremde, der in dem Moment, wo er auf das Gesetz trifft, zum Gast wird, Gegenstand einer Gesetzgebung der Einbeziehung analog zu den Gesetzen der Gastlichkeit? Weswegen ist das Gesetz nie restlos anwendbar, warum vermag es nicht alles zu regeln, warum gibt es einen Überschuss? Anders gefragt: Weshalb ist die Gastlichkeit in den Gesetzen nicht aufgehoben? Gesetze - und das umfasst auch die Gesetze der Gastfreundschaft - sind als Ergebnisse einer Historisierung und Kulturalisierung zu verstehen. Zugleich können sie aber immer nur einen Teil des zu Regelnden vorschreiben - die Schwierigkeit des Gesetzes liegt nicht zuletzt darin, dass es als Allgemeines das Singuläre zu umspannen versucht, und diesem notwendigerweise nicht gerecht zu werden vermag. ${ }^{44}$ Was dies für die Situation des Gastes, zumal des fremden, bedeutet, untersuchen die Beiträge des ersten Schwerpunktes, welche sich der (bereits narrativierten) Spannung zwischen den Gesetzen (Normen, Ritualen, Konventionen) und dem Gesetz der Gastlichkeit (gewissermaßen als ethischem Imperativ) widmen.

\section{Politik}

Mit der Entstehung des neuzeitlichen Nationalstaates, in welchem Territorium und Sprache zur Deckung gebracht werden sollen, wird das frühere ius hospitalis (Gastrecht), das jedem Einwohner zukam, zu einem staatsmonopolistischen Fremden- und Ausländerrecht. Gastlichkeit zerfällt in die private eines persönlichen Gastgebers und in die öffentliche des Landesgastes. Im Zuge einer zunehmend Einheitlichkeit suggerierenden Nationalstaatlichkeit der Moderne verkommt der Gast zum Begriff einer restriktiven Ausländerpolitik, die kulturell-wirtschaftliche Kohärenz mittels territorialer Überwachung und Ausgrenzung gewinnt. Die Realpolitik kanalisiert die notwendigen Arbeitskräfte räumlich und zeitlich unter dem Vorzeichen des

42 Vgl. Waldenfels: Grenzen der Normalisierung, S. 264.

43 Historisch betrachtet, das positivierbare Gastrecht bzw. stellvertretende Handlungen (vgl. Anm. 9).

44 Vgl. Derrida: Gesetzeskraft. 
$>$ Gastarbeiters<. Als >Gastgeberland< setzt man so lange auf die Befristung des Aufenthalts, bis man sich gezwungen sieht, den Gast, der inzwischen schon lange sesshaft geworden ist, mit dem Recht des Daueraufenthalts zu versehen. Die Pejorisierung in Form der so genannten Ausländerpolitik erlaubt die Fortsetzung der Arbeitskräfterekrutierung, bei welcher sich der Staat nur noch die qualifizierten Berufsgattungen herbeiwünscht und die von der Ökonomie dringend benötigten Hilfskräfte in die Illegalität treibt. ${ }^{45}$ Nicht alle >Gäste< sind folglich gleichermaßen willkommen - mit dem Effekt, dass die Politik die vermeintliche Differenz zwischen innen und außen, zwischen eigen und fremd topographiert. Mentale Muster zwischen Gastfreundschaft und Ausgrenzung übertragen sich auf die Landkarte, bestimmen das persönliche Schicksal aufgrund einer Unterscheidung, die letztlich dem Machterhalt und der Besitzstandwahrung dessen dient, der Gesetze aufstellen und durchsetzen kann.

Doch Politik der Gastlichkeit meint gerade das, was nicht im Ausländergesetz verankert ist; denn Politik ist mit Derrida gegen das Gesetz zu lesen. Es handelt sich um eine Politik der Gastfreundschaft, in der Freundschaft politisiert wird. ${ }^{46}$ So lässt sich Gastfreundschaft durchwegs auch als utopisches Gegenkonzept zu einer heutigen Realpolitik der Ausgrenzung begreifen. Dabei darf man sich den durchwegs lapidaren Befund zunutze machen, dass die Gastfreundschaft im gängigen Sprachgebrauch immer noch positiv und die Ausgrenzung negativ konnotiert ist. Im Vordergrund dieser Annäherung an die politische Dimension - jenseits jeglicher Euphemisierung - steht der im Verhältnis zur Gastfreundschaft neutralere Begriff >Gastlichkeit< und das im Gegensatz zur Ausgrenzung prozessgebundene >Liminalitätskonzept<, das der Gast nicht einfach als Fremder, sondern als Gegenüber geradezu einfordert, indem vermeintlich festgesetzte Grenzen immer wieder neu verhandelt und verschoben werden.

In den heute globalisierten Migrationsströmen - welche sich immer zum persönlichen Schicksal kondensieren - gilt es, zunächst den Blick für kulturell-topographische Diskurse zu schärfen, auf deren Folie die literarische Bewertung, Verwertung und Umwertung stattfindet. So richtet sich das Augenmerk im >Bollwerk Europa< gezwungenermaßen vermehrt auf seine institutionalisierten Grenzen, auf seine vermeintlichen kulturellen Ränder und damit auf die topographisch-politischen Schwellen. ${ }^{47}$ Es handelt sich im Kontext einerseits von Globalisierung, andererseits von Regionalisierungstendenzen (innerhalb und außerhalb eines Schengen-Europas) vor allem um diskursive Muster, die zu typischen topographischen Zentralisierungsbemühungen, zur Ausgrenzung oder zur Gastfreundschaft führen und deren Dynamik sowohl produkti-

45 Vgl. dazu Viel Glück! Migration heute.

46 Liebsch: Für eine Kultur der Gastlichkeit, S. 143-177; sowie Derrida: Politik der Freundschaft.

47 Vgl. dazu den Beitrag von Boris PREVIŠIĆ in diesem Band. 
ons- wie rezeptionsästhetisch aufgenommen, weiterentwickelt und unterminiert wird. ${ }^{48}$ Der Raum in seiner topographischen Funktionalisierung oszilliert so zwischen eindeutiger Zuschreibung und Hybridisierung, zwischen Metaphorisierung und Metonymisierung. Je klarer die Grenzen und somit die Ausgrenzungsmechanismen festgelegt werden, je klarer die Diskurshoheit über einen geographischen Raum behauptet wird, desto stärker sind auch die Gegenbewegungen.

Die beiden Phänotypen Ausgrenzung und Gastfreundschaft standen noch nie zuvor in einem so eklatanten Widerspruch wie heute: Einerseits erzwungene Migration, die als politisch induzierte meist noch auf Akzeptanz stößt, der als ökonomische oder neuerdings ökologische hingegen Schmarotzertum unterstellt wird, andererseits freiwillige Mobilität, die meist positiv bewertet wird. $\mathrm{Zu}$ fragen wäre, ob der literarische Diskurs im Zeichen der Gastlichkeit neue Modelle jenseits einer ökonomischen Dichotomisierung bereitstellt. Das so genannte Gastland hält unterschiedliche Aufnahme-Modelle bereit, welche von vollständiger Ausgrenzung (man denke nur an >Gastarbeiter $<$ auf der arabischen Halbinsel) über Assimilation (von Sprache, Werten oder sogar Religion) bis zu einer fruchtbaren Orientierung an der Andersartigkeit reichen. Inwiefern könnte Literatur ohne vermeintlich kulturell grundierte Differenzen überhaupt noch erzählen?

Doch bevor der Anschluss an literarische Konzepte oder an die Fingierung gastlicher Urszenen gesucht werden kann, muss die Anknüpfung sowohl an Ausgrenzungsdiskurse und -erfahrungen in politischen Kontexten als auch an eine philosophisch-ethisch induzierte Debatte zu problematischen und politisierten Stichworten wie Identität oder Integration, welche der realpolitische Kontext als Denkfiguren aufgibt, vorgenommen werden. ${ }^{49}$

\section{Erzählung}

Wie hängen Erzählung - und damit auch Sprache - mit Gastlichkeit zusammen? Warum wird der Gast narrativiert, weshalb und wovon wird im Rahmen der gastlichen Begegnung erzählt? Um sich der narrativen Dimension des Gastes bzw. der Gastlichkeit anzunähern, muss das Verhältnis von Sprache zum Empfang des Gastes, seiner Rede oder Wortlosigkeit $^{50}$ untersucht werden. Zunächst gilt es, sich dem grundsätzlichen Verhältnis von Gast(lichkeit) und Sprache anzunähern, und zwar als einem reziproken: als Sprache in der Gastlichkeit und Gastlichkeit in der Sprache.

48 Vgl. dazu den Beitrag von Svjetlan LACKO VIDULIĆ in diesem Band.

49 Hier schließt der Beitrag von Burkhard LIEBSCH an.

$50 \mathrm{Vgl}$. dazu den Beitrag von Rolf PARR in diesem Band. 
Gesprochene Sprache - und damit auch Erzählung - sind in der gastlichen Aufnahme scheinbar nicht im selben Ausmaß Voraussetzung von Gastlichkeit wie Bewirtung und Unterbringung, die von lebensnotwendiger Bedeutung sein können. Dennoch stellt Sprache hier insbesondere als Gesprochenes - Bestandteil der gastlichen Situation dar, mindestens aber in Form von Gestik, Mimik u.ä. Ausgebildete Sprachen erweitern also von vornherein die Sphären gastlicher Möglichkeiten. Innerhalb dieses >Überschusses <, d.h. der Erweiterung gastlicher Teilnahme durch die Sprache, kann sie zunächst als Teil der gastlichen Gesetze betrachtet werden, beispielsweise als Instrument im Rahmen der Verpflichtung, sich auszuweisen bzw. sich durch Nennung des Namens zu erkennen zu geben; gemäß dieser Auffassung würde die Funktion von Sprache darin bestehen, Sicherheit zu schaffen, die potenzielle Beunruhigung durch den Gast zu vermeiden, indem beispielsweise eine Identifikation des Ankommenden sprachlich gewährleistet wird, welche die Erzählung des Gastes von sich selbst, seines Namens, seiner Herkunft, seiner Absichten und Ziele enthält.

Innerhalb der gastlichen Begegnung tritt andererseits neben der informativ-biografischen Absicht zur Feststellung der Identität gehäuft das Erzählen als solches in den Mittelpunkt - sei es als Erzählen von Erfahrungen (z.B. im Sinne eines Reise- oder Erlebnisberichts), sei es als Erzählung zur Unterhaltung des Gastes oder des Gastgebers, die den Charakter einer Gabe annimmt. Auch in letzterem Falle stellt die Sprache Teil einer Überhöhung, einer Ausweitung gastlicher Verhältnisse dar, die sich im Umfeld des Gastes wiederholt findet.

Sprache ist demnach traditionell immer bereits in die gastliche Situation eingebettet, doch vermag sie die vermeintliche Beruhigung, die insbesondere ihrem funktionalen Verständnis innewohnt, nicht letztgültig herzustellen: Denn Sprache als Medium der Erfahrbarkeit und Mittel des Ausdrucks ist weit entfernt von einer bloßen Funktionsaufgabe. ${ }^{51}$ Sie ist weder $\mathrm{zu}$ beherrschen, noch kann man ihr einfach zudienen, womit sie in einem eigenartigen und schillernden Analogieverhältnis zur Gastlichkeit selbst steht: dem Gast als »ungegebene[m] Dritte[n] « ${ }^{52}$ gesellt sich - mit den Worten Hans-Jost Freys - »die Sprache [als] das Dritte « hinzu. ${ }^{53}$ Der sprachliche Überschuss, der sich der umfassenden Beherrschung entzieht, zeigt sich beispielsweise in Adalbert Stifters

51 Die Differenz, welche in Absetzung von einer Alltagssprache in Bezug auf die literarische Sprache gemacht wird (vgl. Mukařovský: »Standard Language and Poetic Language «), wird ebenfalls in der literarischen Fiktion unter dem Vorzeichen künstlerischer Rahmung generiert (vgl. Lotman: Die Struktur literarischer Texte). Das Setting von Rahmen- und Binnenerzählung ist gerade für das Arrangement der Gastlichkeitsszene von zentraler Bedeutung. Vgl. dazu den Beitrag von Alexander HONOLD in diesem Band. 
späten, ritualhaft überformten Erzähltexten, welche Ordnungen suggerieren, in denen sich dennoch - oder gerade deswegen - Unwägbarkeiten einschleichen, welche die Ordnung als künstliches und labiles Konstrukt entlarven. ${ }^{54}$

Der vermeintlichen Sicherheit des Für-sich-selbst-Zeugens des Gastes ist zudem bereits ein grundlegender Zweifel eingeschrieben: Zeugenschaft durch Sprache ist problematisch, insbesondere, da der Zeuge seiner selbst keinen eigenen Zeugen kennt, womit der Fiktionalitätscharakter im Falle der Identifikation, Absichtserklärung u.s.w. eines Gastes nicht gänzlich ausgeschlossen werden kann. Die sprachliche Äußerung, das sprachliche Zeugnis, das als Akolyt (Begleiter und Zeuge) der Person dienen soll, kann sich genauso als Anakoluth herausstellen, wie dies z.B. in Kleists »Die Verlobung in St. Domingo« der Fall ist. Gerade im Falle des für sich selbst zeugenden Gastes wird deutlich, dass letztgültige Sicherheit nicht möglich ist; an ihre Stelle muss der Glaube, das Vertrauen treten, das jedoch nicht als Pakt geschlossen werden kann, sondern als gastfreundliche Gabe gegeben werden muss. ${ }^{55}$

Aus dem prekären Sprachverhältnis der Literatur, in der sich keine realweltliche Referenz zur sprachlichen Zeugenschaft gesellen kann, ergibt sich nicht zuletzt auch die Relevanz der Gastlichkeit aus theoretischer Perspektive. Eine potenzierte Wahrnehmung dieses Status ermöglicht die Betrachtung literarischer Sprache, deren Mangel an einer literarischen Essenz sich zugleich mit dem Überschuss, dem Einbruch der Sprache (bzw. des Zeichens) in die Sprache selbst kreuzt: Das Ankommen des Gastes wird in der Literatur immer schon als sprachliches Ereignis inszeniert. ${ }^{56}$ Wenn die Sprache als Medium der Gastlichkeit verstanden wird, inwiefern ist dann die gastliche Situation per se mit der Generierung von Narration verknüpft? Lässt sich aus der Situation des ankommenden Gastes, die zum Erzählen führt, die Erzählung zugleich als Gabe und performatives Ereignis denken?

Narratologisch und motivisch ergeben sich daraus folgende Konsequenzen: Die den literarischen Texten inhärenten Grammatiken des Gastes, dessen Rolle sich zwischen Abweisung und Aufnahme definiert, stellen sämtliche dichotomisch angelegten Oppositionen in Frage. Der Gast als Figur des Dritten eröffnet erzählstrategische Möglichkeiten, erscheint er selbst doch nur an der Schwelle, an der ihm ein Sprach-Raum eröffnet wird. Narratologisch ließe sich die Schwelle als Beginn eines jeden literarischen Ereignisses und somit der

54 So z.B. Koschorke/Ammer für Stifters »Der fromme Spruch« (vgl. dies.: »Der Text ohne Bedeutung oder die Erstarrung der Angst «).

55 Vgl. Derrida: Eine gewisse unmögliche Möglichkeit.

56 Vgl. ebd., S. 33f. 
Erzählung lesen. ${ }^{57}$ Welche strukturellen Folgen ergeben sich für die Schwelle als Handlungstopographie im Hinblick auf die Narrationsbildung literarischer Texte ${ }^{58}$

$»[D]$ er die Ein- und Ausschlüsse durchquer[ende] ${ }^{59}$ Gast erweist sich als primäre Figur von Liminalität; eingefasst in narrative Modi, erzeugt er eine mehrschichtige Beobachtungsstruktur, welche Literatur in vielfacher Form hybrid werden lässt. Geht man davon aus, dass das Gastsein auch anthropologisch die Voraussetzung jeglicher Kultur bildet, um in der ephemeren Aufnahme des Fremden das (immer durch den Inzest und die Inzucht bedrohte) Eigene aufzufrischen und damit zu perpetuieren $^{60}$, so erzählt die Literatur nicht einfach von der Begegnung, sondern ebenso von ihrer eigenen Existenz, indem sie ihr Erzählen selbst thematisiert und perspektiviert. In dieser doppelten Kodierung spricht sie immer von menschlicher Genealogie und handelt gleichzeitig in Selbstbeobachtungskategorien, welche sich in unterschiedlichen narrativen Modi niederschlagen. So gewinnt die gastliche Szene des Gedichts beispielsweise in ihrer Narrativierung an Dynamik. Die Fatalität, welche sich aus einer reinen Selbstbezüglichkeit der Kultur und ihrer Erzählung ergibt, wird mit dem Einlass des Fremden aufgebrochen. Erst die intra- und intermediale Hybridisierung des Mediums selbst, die Ausreizung der Differenzen zwischen Schriftlichkeit und Mündlichkeit, zwischen Poesie und Prosa, zwischen Rahmentext und Binnenerzählung, zwischen Erzählung und poetologischer Reflexion, zwischen Plot- und Erzählvorgang sowie zwischen unterschiedlichen Erzählgenres ermöglicht eine Beobachtung zweiter Ordnung, welche wiederum eine Fortsetzung narrativer Muster und weiterer Kulturgenerationen erlaubt. ${ }^{61}$ Sowohl in phylo- wie in ontogenetischer Hinsicht auf die Kultur wie auf ihr Erzählen selbst entsteht ein Zusammenhang zwischen Genre, Genealogie und Reproduktion.

In der narrativen Verhandlung des Themenkomplexes von Gastfreundschaft und Ausgrenzung stehen daher Familiengeschichten immer wieder im Zentrum, da sie gleichsam den kulturellen Nukleus bilden, wie es die einschlägigen Textbeispiele in diesem Band zeigen, beispielsweise die Genealogie zwischen Gesetz und Ausnahmezustand in Stifters »Der Kuß von Sentze ${ }^{62}$ oder in der bereits erwähnten

57 Vgl. Lotman: Die Struktur literarischer Texte und, spezifisch für die gastliche Szene, Simon: »Ikononarratologie«.

58 Vgl. dazu Ralf Simons Beitrag in diesem Band.

59 Bahr: Die Sprache des Gastes, S. 15.

60 Vgl. Lévi-Strauss: Die elementaren Strukturen der Verwandtschaft, S. 57.

61 Darüber, inwiefern gerade Literatur systemtheoretisch gedacht werden kann, gibt folgende Monographie den umfassendsten Überblick: Sill: Literatur in der funktional differenzierten Gesellschaft.

62 Vgl. Evi FountoulaKIS’ Beitrag in diesem Band. 
Erzählung Kleists »Die Verlobung in St. Domingo ${ }^{63}$. Ob dabei intertextuelle Anlagen (vor allem mit einschlägigen ödipalen Bezügen) als Gefahr oder Chance gewertet werden, ist erst dem konkreten Hypotext zu entnehmen. So erweist sich die scheinbar unabwendbare Tragik in Štiks' Roman Die Archive der Nacht, welche schon sehr früh im Roman mit Frischs Homo Faber unterminiert und von unbewältigten Gespenstern der Vergangenheit eingeholt wird, lediglich als Metonymie für die Schicksalsergebenheit Europas angesichts seines Scheiterns in den Sezessionskriegen Jugoslawiens. ${ }^{64}$ Damit artikuliert sich in den narrativen Mustern eine potentielle Utopie, welche auch auf einen gesellschaftspolitischen Zusammenhang verweist. ${ }^{65}$ Die schwer zu fassende performative Funktion der Sprache scheint sich aber, wie einige Beiträge aufzeigen, in literarischen Texten zugleich der strukturellen Formalisierung zu entziehen.

Der literarische Text der Gastlichkeit erzeugt in seinem Zwang zur Selbstbeobachtung neue Kategorisierungsebenen: Aus dem intrinsischen Verhältnis von Sprache, Erzählung und Gastlichkeit existiert zunächst eine Ebene, auf welcher die Erzählung, welche von der Gastlichkeit handelt, selber zu Gast ist, so zum Beispiel im Verhältnis von Binnen- zu Rahmenerzählung. Dass Gastlichkeitserzählungen geradezu auf zusätzliche Binnenerzählungen angewiesen sind, lässt sich an den bereits genannten literarischen Werken eindrücklich aufzeigen.

\section{Sprache der Gastlichkeit, Gastlichkeit der Sprache}

Da die Literatur mit ihrem Fundus an Anschauungsmaterial von Erzählmustern über ein immenses kulturelles Gedächtnis verfügt ${ }^{66}$ und eine wesentliche Rolle als Bindeglied zwischen Theorie und Realitätsreferenz (nicht zuletzt auch im Sinne literarischer >Fallstudien<) einnimmt, konzentriert sich dieser Band auf strukturelle und historische Aspekte, um so die narrative Alterität in der Literatur in der ganzen methodischen und theoretischen Breite analytisch und jeweils exemplarisch dingfest zu machen.

Dass die Gastlichkeit insbesondere dort ein bemerkenswertes Phänomen für die Literatur darzustellen scheint, wo sie problematisiert

63 Vgl. den Beitrag von Csongor LÖRINCZ in diesem Band.

64 Vgl. Milka CARS Beitrag in diesem Band.

65 Diesen Modus der spezifischen Figurenlektüre nennt Said kontrapunktische Lektüre (vgl. Said: »Reflections on Exile«), welche Dunker in seiner Monographie fruchtbar zu machen versucht (vgl. Dunker: Kontrapunktische Lektüren).

66 Es geht hier also nicht einfach um die pure Anhäufung einer historischen Faktizität im Laufe der Zeit als vielmehr um die Modulierung, Strukturierung und erzählte Verfügbarkeit von Geschichte. Einschlägig dazu: Assmann: »Erinnerung als Erregung. Wendepunkte der deutschen Erinnerungsgeschichte«. 
oder verweigert wird und dementsprechend in Ausgrenzung mündet, ist eine Beobachtung, die zahlreiche der vorliegenden Beiträge machen. Die Gastlichkeit als ökonomischer Tauschhandel, wie ihn zahlreiche Studien thematisieren ${ }^{67}$, der Tausch als fraglos wesentlicher Aspekt der konventionellen Handlungen der gastlichen Begegnung bis hin zur umfassenden Ökonomisierung bzw. Industrialisierung der Gastfreundschaft im Tourismuswesen, wird paradoxerweise immer zugleich hyperbolisch von der Gabe überhöht. ${ }^{68}$ Der Gastlichkeit scheint die >hyperbolé< schon immer innezuwohnen, besteht sie doch bereits von ihrer Grundanlage bzw. ihrem frühesten Verständnis her gerade in der Überschreitung der Gesetze und der Überwindung der bloßen Politik (oder dessen, was heute mehrheitlich darunter zu verstehen ist: eine auf ökonomischem Kalkül basierende Verbindung). Die Literatur bildet einerseits das Medium, das diesen Überschuss nicht nur wiederzugeben, sondern selbst zu performieren vermag. Das ist teilweise ihrem nichtpragmatischen Sprachmodus und dem Fehlen einer für die Literatur spezifischen bzw. ihr inhärenten Essenz zuzuschreiben. ${ }^{69}$ Andererseits, und hierin gibt sich Sprache als >erste Gastgeberin< zu verstehen, bildet die Sprache den Raum, in der Gastlichkeit statt hat, von der Begrüßung bis zu Verabschiedung, in der ihr ein Raum eröffnet wird, in- und außerhalb der Literatur. Um es in den Worten Levinas zu sagen: »Sprache ist Gastlichkeit . $^{70}$

\section{Literatur}

Assmann, Aleida: »Erinnerung als Erregung. Wendepunkte der deutschen Erinnerungsgeschichten«. In: Wolf Lepenies (Hg.): Jb. Wissenschaftskolleg zu Berlin 1998/99. Berlin: Siedler 2000, S. 200-220.

Bahr, Hans-Dieter: Die Anwesenheit des Gastes. Entwurf einer Xenosophie [erscheint 2010].

Bahr, Hans-Dieter: Die Befremdlichkeit des Gastes. Wien: Passagen 2007.

Bahr, Hans-Dieter: »Die Gastlichkeit des Buches«. In: ders.: Die Befremdlichkeit des Gastes. Wien: Passagen 2007, S. 53-62.

Bahr, Hans-Dieter: Die Sprache des Gastes. Eine Metaethik. Leipzig: Reclam 1994.

Benveniste, Émile: Indoeuropäische Institutionen. Wortschatz, Geschichte, Funktionen. Übersetzt von Wolfgang Bayer, Dieter Hornig und Katerina

67 Aus jeweils unterschiedlichen Perspektiven thematisieren beispielweise folgende Texte das Verhältnis von Tausch und Gabe: Benveniste: Indoeuropäische Institutionen; Derrida: Falschgeld; Mauss: Die Gabe; Starobinski: Gute Gaben, schlimme Gaben.

68 Vgl. Dufourmantelle: »Einladung«, S. 128ff.

69 Vgl. Derrida: »This strange institution called literature«.

70 Levinas: Totalität und Unendlichkeit, S. 444. 
Menke. Frankfurt am Main: Campus 1993. / Le vocabulaire des institutions indo-européennes. Paris: Les Éditions de Minuit 1969.

Bürner-Kotzam, Renate: Vertraute Gäste. Befremdende Begegnungen in Texten des bürgerlichen Realismus. Heidelberg: Winter 2001.

Derrida, Jacques: Eine gewisse unmögliche Möglichkeit, vom Ereignis zu sprechen. Aus dem Französischen von Susanne Lüdemann. Berlin: Merve 2003. / »Une certaine possibilité impossible de dire l'événement «. In: Gad Soussana, Alexis Nouss und Jacques Derrida (Hg.): Dire l'événement, est-ce possible? Séminaire de Montréal pour Jacques Derrida. Paris: L'Harmattan 2001, S. 79-112.

Derrida, Jacques: Falschgeld. Zeit geben I. Übersetzt von Andreas Knop und Michael Wetzel. München: Fink 1993. / Donner le temps. 1. La fausse monnaie. Paris: Galilée 1991.

Derrida, Jacques: Gesetzeskraft. Der »mystische Grund der Autorität«. Übersetzt von Alexander García Düttmann. Frankfurt am Main: Suhrkamp 1996. / Force de loi. Le »Fondement mystique de l'autorité«. Paris: Galilée 1994.

Derrida, Jacques: Politik der Freundschaft. Übersetzt von Stefan Lorenzer. Frankfurt am Main: Suhrkamp 2000. / Politiques de l'amitié. Paris: Galilée 1994.

Derrida, Jacques: »This strange institution called literature «. In: ders.: Acts of Literature. Hg. von Derek Attridge. New York; London: Routledge 1992, S. 33-75.

Derrida, Jacques: Von der Gastfreundschaft. Aus dem Französischen von Markus Sedlaczek. Wien: Passagen 2001. / De l'hospitalité. Anne Dufourmantelle invite Jacques Derrida à répondre. Paris: Calman-Lévy 1997.

Dufourmantelle, Anne: »Einladung«. In: Jacques Derrida: Von der Gastfreundschaft. Mit einer »Einladung « von Anne Dufourmantelle. Hg. von Peter Engelmann. Wien: Passagen 2007, S. 111-144.

Dunker, Axel: Kontrapunktische Lektüren. Koloniale Strukturen in der deutschsprachigen Literatur des 19. Jahrhunderts. München: Fink 2008.

Etymologisches Wörterbuch des Deutschen. Hg. von Wolfgang Pfeifer. 2 Bde. Berlin: Akademie Verlag ${ }^{2} 1993$.

Frey, Hans-Jost: Die Autorität der Sprache. Lana; Wien; Zürich: Edition Howeg + edition per procura 1999.

Friedrich, Peter und Rolf Parr (Hg.): Gastlichkeit. Erkundungen einer Schwellensituation. Heidelberg: Synchron 2009, S. 281-299.

Handke, Peter: Mein Jahr in der Niemandsbucht. Ein Märchen aus den neuen Zeiten. Frankfurt am Main: Suhrkamp 1994.

Hoffmann, E. T. A.: »Der Sandmann«. In: ders.: Sämtliche Werke in sechs Bänden. Hg. von Hartmut Steinecke unter Mitarbeit von Gerhard Allroggen. Bd. 3: Nachtstücke. Klein Zaches. Prinzessin Brambilla. Werke 1816-1820. Hg. von Wulf Segebrecht und Hartmut Steinecke 
unter Mitarbeit von Gerhard Allroggen und Ursula Segebrecht. Frankfurt am Main: Deutscher Klassiker Verlag 1985 (= Bibliothek deutscher Klassiker 7), S. 11-49.

Hoffmann, E. T. A.: »Der unheimliche Gast«. In: ders.: Sämtliche Werke in sechs Bänden. Hg. von Hartmut Steinecke und Wulf Segebrecht. Bd. 4: Die Serapions-Brüder. Hg. von Wulf Segebrecht unter Mitarbeit von Ursula Segebrecht. Frankfurt am Main: Deutscher Klassiker Verlag 2001 (= Bibliothek deutscher Klassiker 175), S. 722-772.

Kleist, Heinrich von: »Die Verlobung in St. Domingo«. In: ders.: Sämtliche Werke. Berliner Ausgabe. Hg. Roland Reuß und Peter Staengle. Bd. II/4. Basel: Stroemfeld / Roter Stern 1988, S. 7-94.

Koschorke, Albrecht und Andreas Ammer: »Der Text ohne Bedeutung oder die Erstarrung der Angst. Zu Stifters letzter Erzählung >Der fromme Spruch «. In: DVjs 61 (4, 1987), S. 677-719.

Kristeva, Julia: Fremde sind wir uns selbst. Übersetzt von Xenia Rajewsky. Frankfurt am Main: Suhrkamp 2008. / Étrangers à nous-mêmes. Paris: Fayard 1988.

Lévi-Strauss, Claude: Die elementaren Strukturen der Verwandtschaft. Übersetzt von Eva Moldenhauer. Frankfurt am Main: Suhrkamp 1993. I Les structures élémentaires de la parenté. Paris: PUF 1949.

Levinas, Emmanuel: Die Spur des Anderen. Untersuchungen zur Phänomenologie und Sozialphilosophie. Übersetzt, herausgegeben und eingeleitet von Wolfgang Nikolaus Krewani. Freiburg im Breisgau: Alber 1983. / Le temps et l'autre. Montpellier: Fata Morgana 1979.

Levinas, Emmanuel: Totalität und Unendlichkeit. Übersetzt von Wolfgang Nikolaus Krewani. Freiburg im Breisgau: Alber 1993. I Totalité et infini. Essai sur l'extériorité. La Haye: Nijhoff 1961.

Liebsch, Burkhard: Für eine Kultur der Gastlichkeit. Freiburg im Breisgau: Alber 2008.

Lotman, Jurij: Die Struktur literarischer Texte. München: Fink 1972.

Mauss, Marcel: Die Gabe. Form und Funktion des Austauschs in archaischen Gesellschaften. Frankfurt am Main: Suhrkamp 2009. I »Essai sur le don. Forme et raison de l'échange dans les sociétés archaïques«. Année Sociologique 1923-1924.

Mukařovský, Jan: »Standard Language and Poetic Language«. In: Paul L. Garvin (Hg.): A Prague School Reader on Esthetics, Literary Structure, and Style. Georgetown: University Press 1964, S. 17-30.

Parr, Rolf und Peter Friedrich: »Von Gästen, Gastgebern und Parasiten«. In.: dies. (Hg.): Gastlichkeit. Erkundungen einer Schwellensituation. Heidelberg: Synchron 2009, S. 7-14.

Peyer, Hans Conrad (Hg.): Gastfreundschaft, Taverne und Gasthaus im Mittelalter. München: Oldenbourg 1983.

Polaschegg, Andrea: Der andere Orientalismus. Regeln deutsch-morgenländischer Imagination im 19. Jahrhundert. Berlin: Gruyter 2006. 
Schumacher, Meinolf: »Gast, Wirt und Wirtin. Konstellationen von Gastlichkeit in der Literatur des Mittelalters«. In: Rolf Parr und Peter Friedrich (Hg.): Gastlichkeit. Erkundungen einer Schwellensituation. Heidelberg: Synchron 2009, S. 105-116.

Simmel, Georg: »Exkurs über den Fremden« (1908). In: ders.: Soziologie. Untersuchungen über die Formen der Vergesellschaftung. $\mathrm{Hg}$. von Otthein Rammstedt. Frankfurt am Main: Suhrkamp 1992, S. 764-771.

Simon, Ralf: »Ikononarratologie. Bildtheoretische Grundlegung der Narratologie in der Szenographie der Gastlichkeit«. In: Alexander Honold und Ralf Simon (Hg.): Das erzählende und das erzählte Bild. München: Wilhelm Fink 2010, S. 303-329.

Sill, Oliver: Literatur in der funktional differenzierten Gesellschaft. Systemtheoretische Perspektiven auf ein komplexes Phänomen. Wiesbaden: Westdeutscher Verlag 2001.

Starobinski, Jean: Gute Gaben, schlimme Gaben. Die Ambivalenz sozialer Gesten. Aus dem Französischen von Horst Günther. Frankfurt am Main: Fischer 1994.

Štiks, Igor: Die Archive der Nacht. Übersetzt von Marica Bodrožić. Berlin: Claassen 2008. / Elijahova stolica. Zagreb: Fraktura 2006.

Stifter, Adalbert: »Der Kuß von Sentze«. In: ders.: Werke und Briefe. Historisch-kritische Gesamtausgabe. Herausgegeben von Alfred Doppler und Hartmut Laufhütte. Bd. 3,2. Stuttgart: Verlag W. Kohlhammer 2003, S. 141-174.

Viel Glück! Migration heute. Wien, Belgrad, Zagreb, Istanbul. Hg. von Vida Bakondy, Simonetta Ferfoglia, Jasmina Janković, Cornelia Kogoj, Gamze Ongan, Heinrich Pichler, Ruby Sircar und Renée Winter. Wien: Mandelbaum 2010.

Waldenfels, Bernhard: Grenzen der Normalisierung. Frankfurt am Main: Suhrkamp 2008.

Waldenfels, Bernhard: Topographie des Fremden. Frankfurt am Main: Suhrkamp 2003.

Waldenfels, Bernhard: Verfremdung der Moderne. Göttingen: Wallstein 2001. 
Bereitgestellt von | Universitaetsbibliothek Basel

Angemeldet

Heruntergeladen am | 09.10.18 10:32 